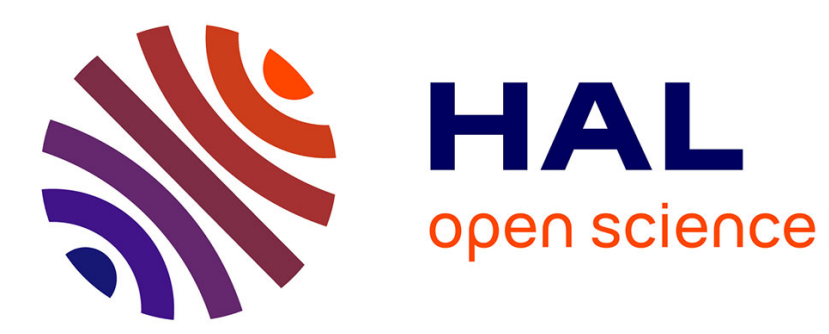

\title{
Influence of moderate pressure nanosecond discharge on the structure of detonation wave
}

\author{
Mhedine Ali Cherif, Sergey Shcherbanev, Pierre Vidal, Alain Claverie, \\ Svetlana Starikovskaia
}

\section{- To cite this version:}

Mhedine Ali Cherif, Sergey Shcherbanev, Pierre Vidal, Alain Claverie, Svetlana Starikovskaia. Influence of moderate pressure nanosecond discharge on the structure of detonation wave. AIAA Scitech 2020 Forum (Session: Plasma Assisted Combustion II), Jan 2020, Orlando, United States. $10.2514 / 6.2020-1894$. hal-03005023

\section{HAL Id: hal-03005023 \\ https://hal.science/hal-03005023}

Submitted on 6 Jan 2021

HAL is a multi-disciplinary open access archive for the deposit and dissemination of scientific research documents, whether they are published or not. The documents may come from teaching and research institutions in France or abroad, or from public or private research centers.
L'archive ouverte pluridisciplinaire HAL, est destinée au dépôt et à la diffusion de documents scientifiques de niveau recherche, publiés ou non, émanant des établissements d'enseignement et de recherche français ou étrangers, des laboratoires publics ou privés. 


\title{
Influence of moderate pressure nanosecond discharge on the structure of detonation wave
}

\author{
Mhedine Ali Cherif* \\ CNRS, Ecole Polytechnique, Sorbonne University, University Paris-Sud, Observatoire de Paris, University Paris-Saclay, \\ Palaiseau, 91128, France \\ Sergey A. Shcherbanev ${ }^{\dagger}$ \\ CNRS, Ecole Polytechnique, Sorbonne University, University Paris-Sud, Observatoire de Paris, University Paris-Saclay, \\ Palaiseau, 91128, France \\ Pierre Vidal ${ }^{\ddagger}$ and Alain Claverie ${ }^{\S}$ \\ Institut Pprime, UPR 3346 CNRS, ENSMA, 86034 Chasseneuil-du-Poitou, France \\ Svetlana M. Starikovskaia ${ }^{\mathrm{I}}$ \\ CNRS, Ecole Polytechnique, Sorbonne University, University Paris-Sud, Observatoire de Paris, University Paris-Saclay, \\ Palaiseau, 91128, France
}

\begin{abstract}
The effect of a volumetric nanosecond discharge on detonation cell size was demonstrated experimentally in a detonation tube test rig. The experiments were performed in $\mathrm{CH}_{4}: \mathrm{O}_{2}: \mathrm{Ar}=1: 2: 2$ mixture, at initial pressure $180 \mathrm{mbar}$ and ambient temperature. The detonation wave was initiated in a 3.6-m long, $50 \times 50-\mathrm{mm}^{2}$ square cross section tube, and entered the measuring section where the electrode system was installed to produce a double-pulse discharge ahead of the detonation front. The triggering of the discharge was synchronized with the arrival of the detonation front to the diagnostic chamber. The plasma was generated by two consecutive pulses of -50 and $-32 \mathrm{kV}$ amplitude on the high-voltage electrode and $25 \mathrm{~ns}$ pulse duration. It was shown that the plasma fills the entire interelectrode space. The analysis of the detonation cell size with and without plasma generation was performed via sooted-plate technique. Production of atoms and radicals in the discharge triggered combustion chemistry decreasing the ignition delay time. As a result, the detonation cell size was reduced by a factor of $1.5-2$, while passing through the region of the discharge.
\end{abstract}

\section{Introduction}

Detonability can be defined as the capacity of detonation to propagate in an explosive substance under specific geometric conditions of confinement. Detonation fronts in homogeneous gases have a cellular structure [1], and a commonly-accepted criterion for self-sustained detonation propagation is that the transverse size of the channel that confines the gas is roughly larger than 3-5 times the mean width of the detonation cell. Mixtures with smaller cells thus have larger detonability. The detonation cell size depends on initial pressure, temperature and composition of the mixture, and the mean width of a detonation cell is considered as a measurement of detonability.

Safety requirements in technological applications imply the use of mixtures with low detonability. In order to avoid self-sustained detonation, the transverse size of the channel should be smaller than some critical value. In contrast, using detonation at conditions close to the self-sustained regime necessitates large channel sizes. Detonation mitigation and enhancement are thus fundamental issues with potential technological applications, such as power generation and propulsion. The ideal detonation process has a higher thermodynamic efficiency than isobaric combustion, a feature that has been motivating a large amount of studies on Pulsed [2] or Rotating [3] Detonation Engines (PDE and RDE, resp.). A detonation wave can be obtained by direct-initiation or deflagration-to-detonation transition (DDT) processes. DDT

\footnotetext{
* Graduate student, Laboratory of Plasma Physics, CNRS, Ecole Polytechnique.

$\dagger$ Postdoctoral researcher, CAPS Laboratory, ETH Zurich.

${ }^{\dagger}$ Sernior researcher, CNRS, Institut Pprime.

$\S$ Research engineer, CNRS, Institut Pprime.

"ISenior researcher, CNRS, Ecole Polytechnique.
} 
length and time are crucial parameters for PDE applications [4] and safety. Several physical and chemical factors influence the DDT parameters [5], one of the most important being the mixture detonability. The cellular detonation structure has been long-time identified both experimentally and numerically [1] as resulting from the interaction of transverse and longitudinal waves that form the detonation leading shock. A well-known experimental fact is that the cell mean width $\lambda$ decreases with increasing initial pressure at constant initial temperature. However, increasing initial pressure can be non-compatible with safety operating conditions. Considering that $\lambda$ is roughly proportional to the characteristic length of the one-dimensional average detonation reaction zone[6], as defined by the Zel'dovich-Von Neuman-Doring (ZND) detonation model, another solution is to change chemical reactivity of the initial mixture so that the reaction length behind the detonation leading shock decreases.

A possible way to enhance chemical reactivity is to produce atoms and radicals by means of non-equilibrium plasma. Energy branching as a function of a mean electron energy in combustible mixtures and changing of the efficient activation energy is discussed in recent reviews [7, 8]. In the field of plasma-assisted combustion, decrease of the ignition delay [9], extending the flammability limits and increasing the combustion stability of lean mixtures [8, 10] have been demonstrated both experimentally and numerically. Shock-tube experiments showed 3-4 orders of magnitude reduction in ignition delay for methane-based mixtures [11] due to dissociation in discharge and partial reforming of the mixture in near afterglow.

Enhancement of the DDT transition under the action of nanosecond plasma has been already demonstrated in $\mathrm{C}_{3} \mathrm{H}_{8}$, $\mathrm{C}_{4} \mathrm{H}_{10}, \mathrm{C}_{6} \mathrm{H}_{14}$ based mixtures [12] and recently in $\mathrm{H}_{2}$ :air mixture [13]. However, the effect of non-thermal plasma on parameters of already formed detonation wave was not investigated before. The ability to decrease the size of the detonation cell by "instantaneously" increasing the reactivity of the gas mixture plays a key role in the problem of detonation control. The aim of the present work is an experimental analysis of the effect of a non-equilibrium plasma on the size of detonation cells. To study this effect, a nanosecond volumetric discharge was initiated ahead of the detonation front in the $\mathrm{CH}_{4}: \mathrm{O}_{2}: \mathrm{Ar}=1: 2: 2$ mixture.

\section{Experimental Setup}

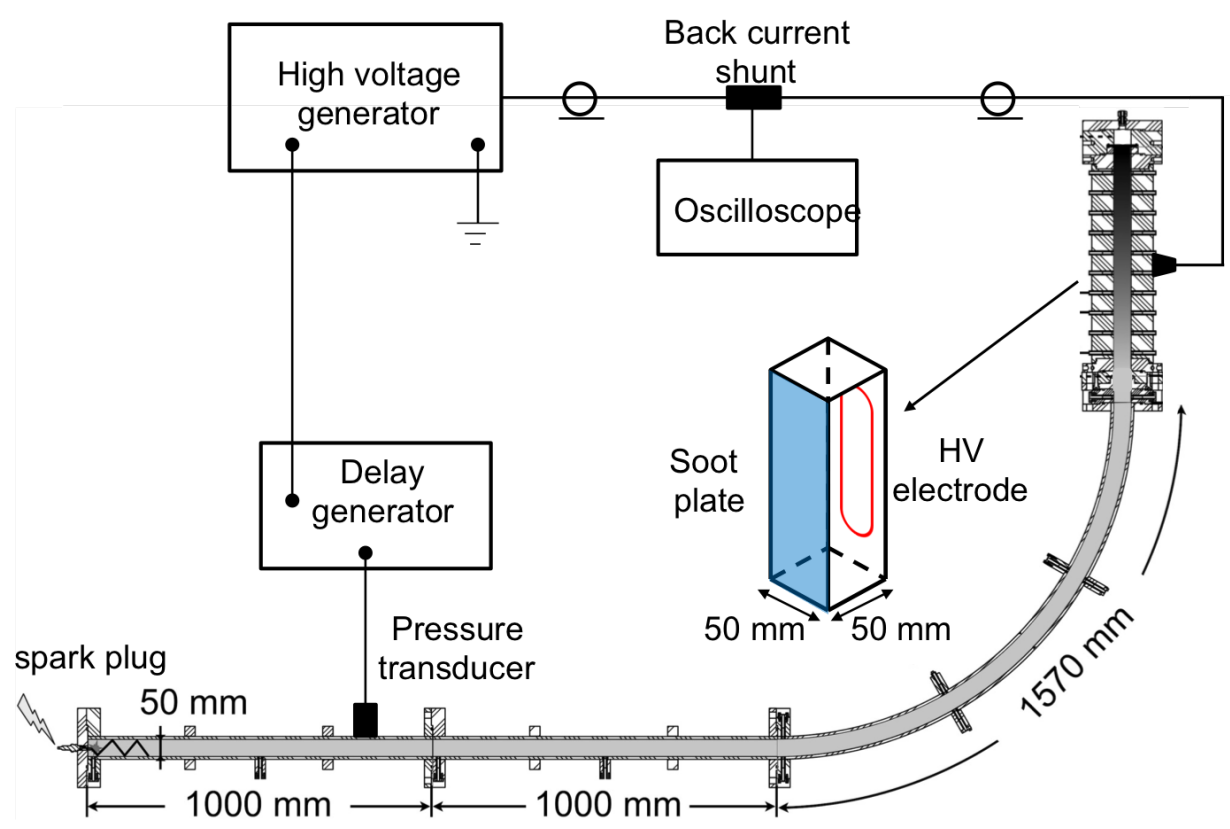

Fig. 1 Schematic of the experimental setup showing the detonation tube and the diagnostic chamber. The locations of the $\mathrm{HV}$ electrode in the diagnostic chamber are shown in red and blue in the insert.

Figure 1 shows a schematic of the experimental setup. The main element is a $665-\mathrm{mm}$ long, $50 \times 50-\mathrm{mm}^{2}$ cross-section vertical diagnostic chamber. The detonation enters the chamber from a $3570-\mathrm{mm}$ long, $50 \times 50-\mathrm{mm}^{2}$ square cross-section detonation tube. The detonation is generated in the tube by means of a DDT process initiated by a spark plug and accelerated by a Shchelkin spiral (Fig 1). 
The squared cross-section diagnostic chamber consisting of four replaceable flanges is shown schematically in the insert in Fig. 1. A brass high-voltage electrode, $25 \times 360 \mathrm{~mm}^{2}$, is installed in a dielectric (PEEK) plate mounted in a stainless steel flange. A sooted plate $\left(1 \mathrm{~mm}\right.$ thick stainless steel plate $50 \times 550 \mathrm{~mm}^{2}$ covered with a thin carbon layer) is mounted on the stainless steel flange opposite to the high-voltage electrode and serves as the grounded electrode. The high-voltage and the grounded electrodes are indicated by red and blue colours, respectively. The other two flanges of the chamber are 500-mm long quartz optical windows.

A set of ten Kistler 603B piezoelectric pressure transducers (response time $1 \mu \mathrm{s}$, natural frequency $300 \mathrm{kHz}$ ) associated with Kistler 5018A electrostatic charge amplifiers (bandwidth $200 \mathrm{kHz}$ ) was used to measure the wave longitudinal mean velocity $D$. The first transducer that detected detonation regime (Fig,1) provided a synchronization signal for triggering the high-voltage generator and the ICCD camera.

A high-voltage generator FPG 25-001NM2C2 (FID GmbH) was designed to deliver a burst of three pulses of negative polarity $10-50 \mathrm{kV}$ on the electrode with 3-4-ns rise time and 25-ns duration at FWHM, with a possibility to change independently the amplitude of each pulse and an adjustable 200-2000-ns delay between them. The high-voltage signal was transmitted through a 30-m long coaxial electrical RG213 cable to the high-voltage electrode. The waveforms of the high-voltage pulses and the deposited energy were measured by means of a custom-made, calibrated back current shunt (BCS) mounted in the grounded shield in the middle of the cable. The BCS was connected to a LeCroy WaveRunner 4Xi-A 600-MHz oscilloscope. The details of the BCS technique have been described elsewhere [14].

The optical emission from the discharge and optical emission produced by the detonation wave was monitored by an ICCD PI-MAX2 camera (Princeton Instruments) with spectral sensitivity range 200-800 nm.

The classical sooted-plate technique was used to compare the structure and the size of the detonation cells obtained without and with plasma action. The sooted plate was changed after each experiment.

\section{Results and Discussion}
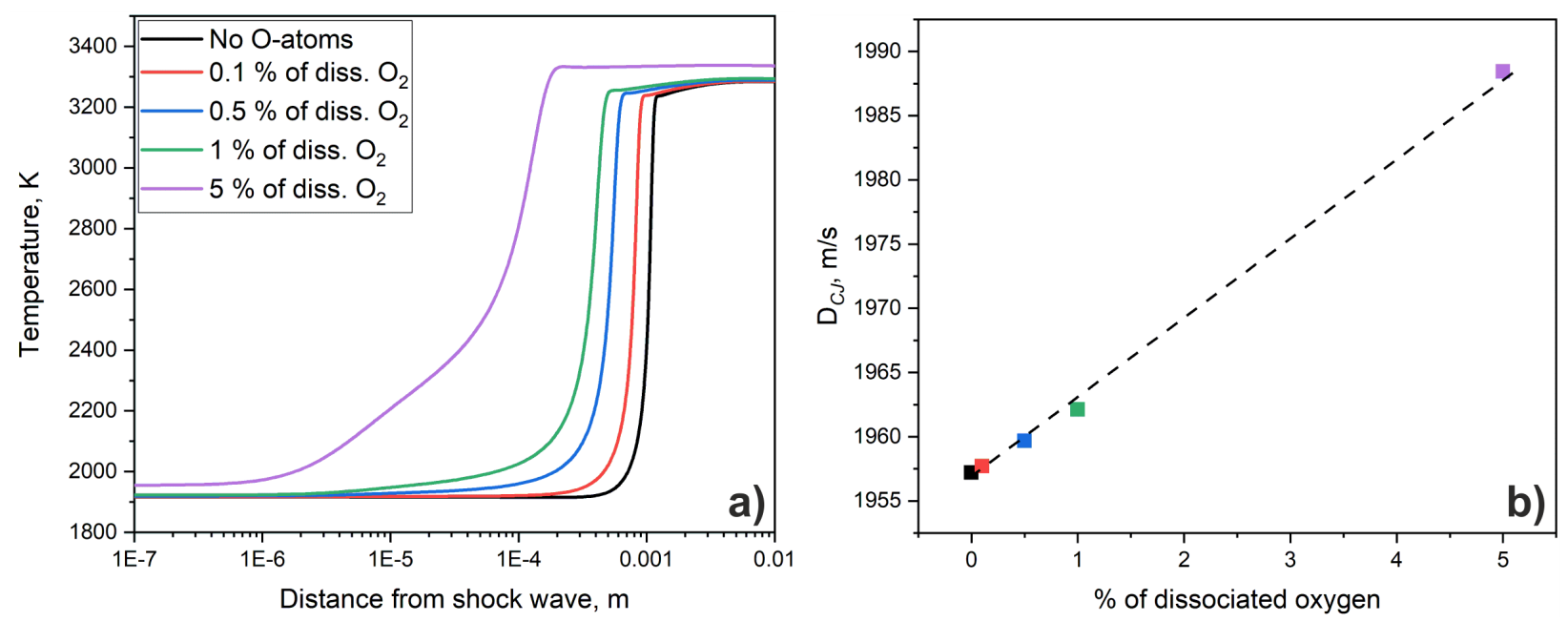

Fig. 2 Calculated a) temperature as a function of distance from shock; and b) Chapman-Jouguet velocity $\left(D_{C J}\right)$ as a function of percentage of dissociated oxygen. The calculations are performed in the $\mathrm{CH}_{4}: \mathrm{O}_{2}: \mathrm{Ar}=1: 2: 2$ mixture for the initial pressure and temperature 180 mbar and $298 \mathrm{~K}$, resp.

The experiments were carried out in a $\mathrm{CH}_{4}: \mathrm{O}_{2}: \mathrm{Ar}$ mixture (1:2:2) at $180 \mathrm{mbar}$ and ambient initial temperature (293-298 K). The detonation cell size is considered as being, in particular, strongly dependent on the activation energy of the mixture, and so on the ignition delay time. The selection of the gas mixture was thus dictated by the fact that methane is the alkane the most sensitive to plasma action, demonstrating the strongest decrease of ignition delay time [9].

A set of preliminary experiments was carried out to study the discharge development in a $\mathrm{CH}_{4}: \mathrm{O}_{2}: \mathrm{Ar}=1: 2: 2$ mixture at ambient temperature and initial pressures ranging from 50 to 200 mbar. The value 180 mbar was found to be optimal to have both a stable detonation and a volumetric plasma.

In order to obtain a qualitative understanding of the plasma action on the detonation wave, the ZND profiles of the 


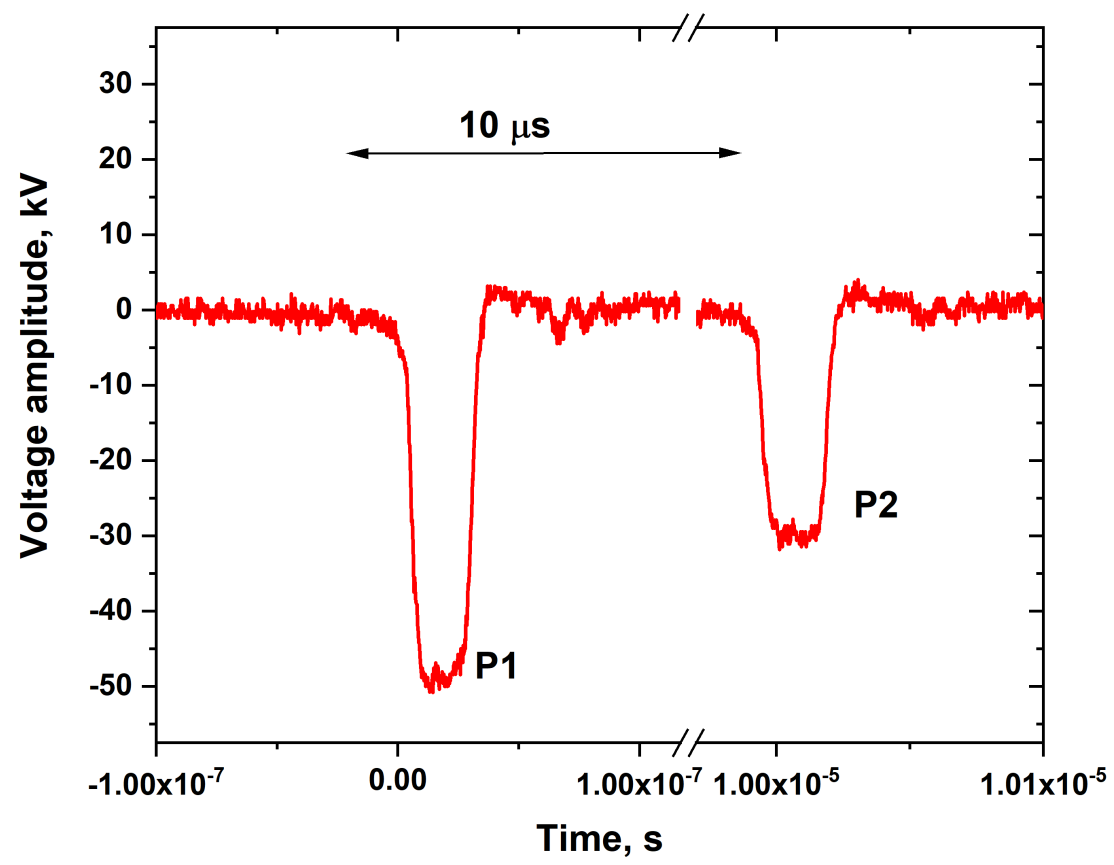

Fig. 3 Voltage waveform on the high-voltage electrode $v s$. time.

average reaction zone were numerically calculated with the Konnov chemical-kinetics mechanism for the considered mixture. Temperature profiles (Fig. 2p) and Chapman-Jouguet (CJ) velocities (Fig. 2p) were calculated with several percentages of dissociated oxygen molecules ( $\%$ of dissociated $\mathrm{O}_{2}$ ) in the mixture ranging from 0 to $5 \%$. The results show that the reaction-zone thickness decreases with increasing $\mathrm{O}$-atoms density: dissociation of about $1 \%$ of $\mathrm{O}_{2}$ reduces the length of the ignition zone (taken at the inflexion point of the temperature profile) by an approximate factor of 2.8. The CJ velocity $D_{C J}$ remains essentially unchanged: $1 \% \mathrm{O}$ increases $D_{C J}$ by $0.25 \%$ only.

2D numerical calculations with detailed chemical kinetics have recently been presented in [15]. The action of a non-equilibrium plasma is modeled by a small amount of radicals locally injected in a hydrogen:air mixture at a certain stage of deflagration-to-detonation transition. The calculations show a cell-size decrease and a faster DDT transition starting from $5 \%$ of atomic oxygen in the mixture.

In the experiments in this work, the amplitude of the high-voltage pulses and the time interval between them were selected to generate a spatially-distributed discharge in the whole domain between the electrodes without transition to arc and without triggering combustion in the mixture. Figure 3 shows the waveform of the high-voltage pulses on the electrode. A sequence of two pulses was selected, with amplitudes on the high-voltage electrode $U=-50 \mathrm{kV}$ for the first one $(P 1)$ and $U=-32 \mathrm{kV}$ for the second one $(P 2)$, and a $10-\mu$ s time interval between them. About $250 \mathrm{~mJ}$ was deposited to the gas mixture in two consecutive pulses, providing a specific deposited energy on the level of parts of $\mathrm{mJ}$ per $\mathrm{cm}^{-3}$ or $10^{-3} \mathrm{eV} /$ particle. At electric fields typical for nanosecond discharge, these values of specific delivered energy result in $\mathrm{O}$-atom densities below $0.1-1 \%$.

ICCD images of the discharge were first taken with a small depth of field $(5 \mathrm{~mm})$ and several focal distances in the direction perpendicular to the longitudinal axis of the discharge section in order to make sure that the discharge is volumetric and fills the central part of the section. Figure 4 a shows ICCD images of the discharge integrated in time during the first pulse. In the considered $\mathrm{CH}_{4}: \mathrm{O}_{2}: \mathrm{Ar}$ mixture at $180 \mathrm{mbar}$, the discharge resulted in a spatially-regular distribution of plasma channels without homogeneity, although in air under the same conditions (pressure, temperature, parameters of the high-voltage pulses) a uniform glow was observed.

The discharge and the detonation wave were synchronized in time. The first discharge was triggered when the detonation wave arrived $5 \mathrm{~cm}$ above the beginning of the high-voltage electrode. Figures $4 \mathrm{p}$ and $\mathrm{c}$ show the detonation 


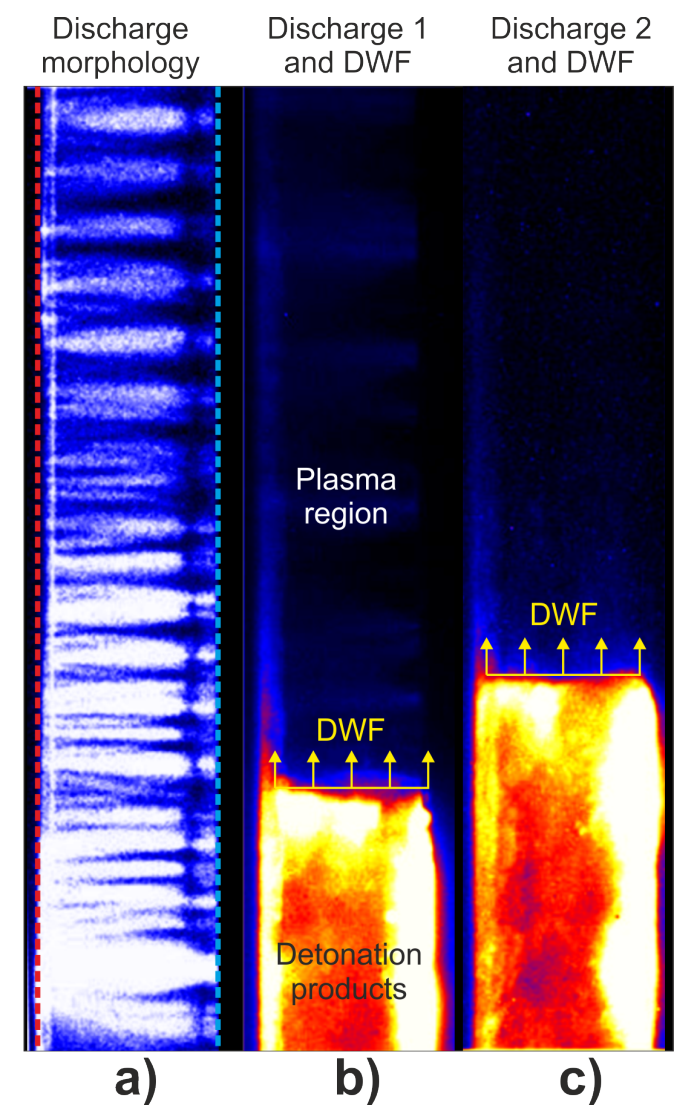

Fig. 4 ICCD images of a) the discharge ignited by the first high-voltage pulse, $P 1$; b) the detonation wave front (DWF) and the discharge at the time corresponding to the initiation of the first discharge pulse $P 1$, time $t_{0}$; c) the detonation wave and the discharge at the time corresponding to initiation of the second discharge pulse $P 2$, time $t_{0}+10 \mu \mathrm{s}$. The gate of the ICCD camera is $500 \mathrm{~ns}$.

wave front (DWF) positions when pulses $P 1$ and $P 2$ were triggered. The intensity of the optical emission from the detonation wave was two orders of magnitude higher than from plasma, so the sensitivity of the images in Figure 4 -a and Figures 4 bc is different.

The detonation velocity measured at the chamber entry is $D=1938.1 \mathrm{~m} / \mathrm{s}$ (the Chapman-Jouguet value is $D_{C J}=1956$ $\mathrm{m} / \mathrm{s}$ [16]). Taking into account the $10-\mu$ s time interval between $P 1$ and $P 2$, the detonation wave thus travels about $2 \mathrm{~cm}$ between the two pulses.

Figure 5 shows typical soot recordings that compare detonation cells without (NP, i.e., no plasma) and with the plasma action (WP, i.e., with plasma). It can be seen that the detonation cell size is reduced through overall the discharge zone. For the NP case, the width $\lambda$ of the larger detonation cells is bounded by the distance $d$ between the chamber walls because of the relatively-low initial pressure (180 mbar), that is, $\lambda$ is equal to $d$. For the WP case, the rightmost three recordings show that $\lambda$ has decreased by a factor about 1.5-2 in the domain P1-EE. Below the P1-P2 domain and above the electrode upper end $(E E)$, that is before and above the plasma zone respectively, $\lambda$ is about equal to the same value $d$ as on the NP recordings.

It should be noted that the time scales of the discharge and the cell-size changing are different. Active species are produced in the discharge, that is, during $P 1$ and $P 2$ pulses, $25 \mathrm{~ns}$ each, and in the near afterglow, at sub-microsecond time scale. The detonation wave passes the region initially occupied by nanosecond plasma during hundreds of microseconds. A possible interpretation is that the radicals produced by the discharge, in particular oxygen atoms, shorten the mixture oxidation time at low pressure and temperature [17]. Plasma thus enhances mixture detonability by providing excited species that enhance reactions behind the detonation front. 


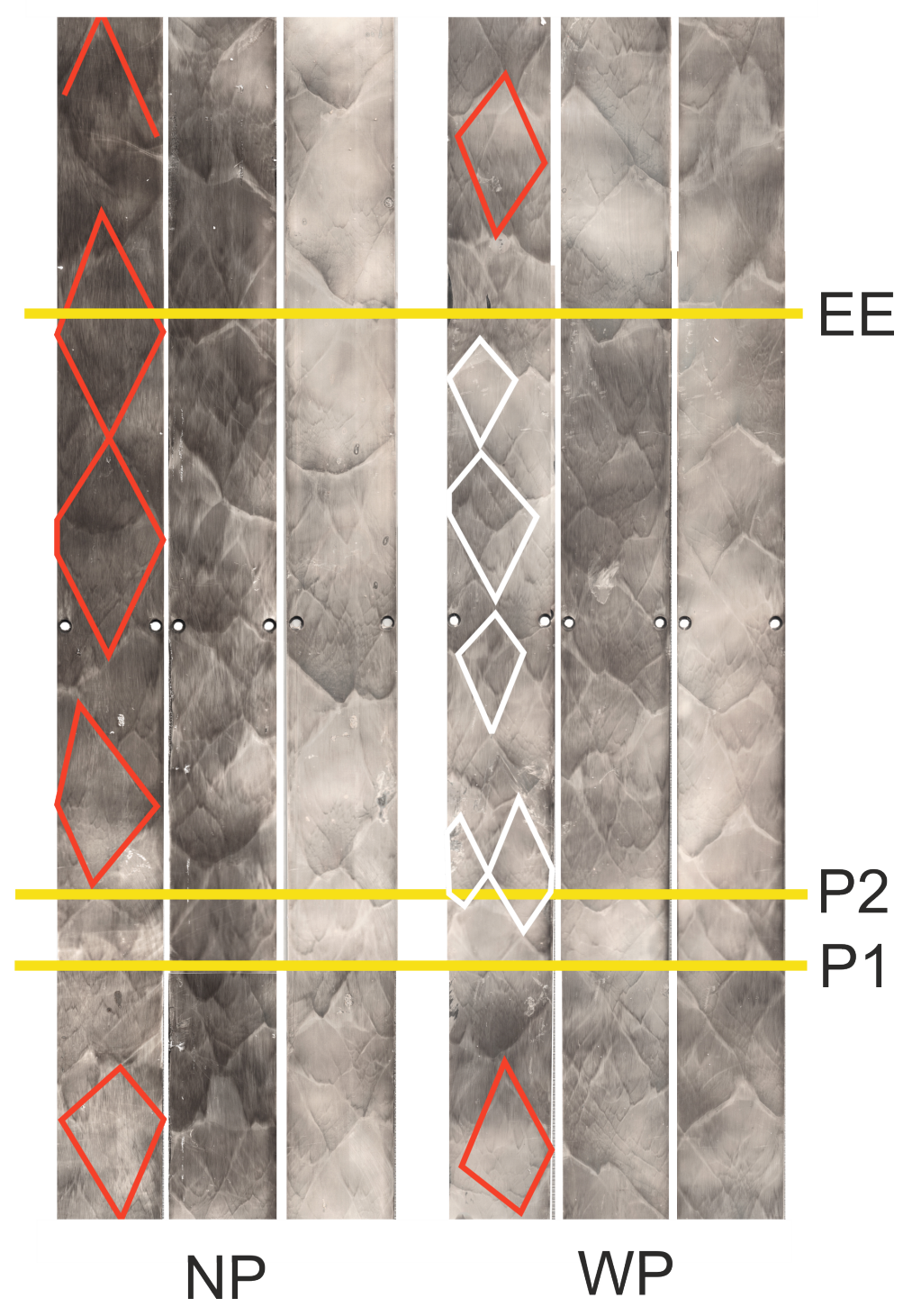

Fig. 5 Soot prints of the detonation wave without plasma (left, NP) and with plasma in the $P 2-E E$ domain (right, WP). Typical sizes of the detonation cells are shown as red and white diamonds.

\section{Conclusion}

In the present work, volumetric nanosecond discharge is obtained in a $\mathrm{CH}_{4}: \mathrm{O}_{2}: \mathrm{Ar}=1: 2: 2$ mixture at $180 \mathrm{mbar}$ and ambient temperature in a $360 \times 50 \times 50-\mathrm{mm}^{3}$ volume ahead of the detonation front. The discharge was initiated by two consecutive pulses of $25-\mathrm{ns}$ duration, and -50 and $-32-\mathrm{kV}$ amplitudes separated by $10 \mu \mathrm{s}$. Use of two pulses allows increasing production of deposited energy without transition to arc discharge. Decrease of detonation cell size by a factor of $1.5-2$ in the overall zone of the discharge is observed experimentally at typical specific deposited energy about $10^{-3} \mathrm{eV} /$ particle. ZND calculations indicates that dissociation of $1 \%$ of molecular oxygen in the considered mixture decreases the ignition zone by a factor 2.8 , but practically does not change the Chapman-Jouguet velocity. It should be noted that the detonation wave passes the region initially occupied by nanosecond plasma during hundreds of microseconds. A suggested interpretation of the observed effect is that plasma triggers the mixture oxidation ahead of the detonation front. 


\section{Acknowledgments}

This work is supported by the convention Ecole Polytechnique-DGA 2790 and by DGA financial support for the $\mathrm{PhD}$ Thesis of Mhedine Ali Cherif. The experiments were carried out at the Pprime institute in Poitiers. The authors wishes to thank Alain Claverie and Maxime Caron for their technical assistances. The electrode system was designed by Ali Majhoub and Jean-Carl Rousseau.

\section{References}

[1] Zhang, F., Shock Waves Science and Technology Library, Vol. 6: Detonation Dynamics, Vol. 6, Springer Science \& Business Media, 2012.

[2] Pandey, K., and Debnath, P., "Review on recent advances in pulse detonation engines," Journal of Combustion, Vol. $2016,2016$.

[3] Anand, V., and Gutmark, E., "Rotating detonation combustors and their similarities to rocket instabilities," Progress in Energy and Combustion Science, Vol. 73, 2019, pp. 182-234.

[4] Frolov, S. M., "Detonation initiation techniques for pulse detonation propulsion," Progress in Propulsion Physics, Vol. 1, 2009, pp. 321-340.

[5] Han, W., Gao, Y., and Law, C. K., "Flame acceleration and deflagration-to-detonation transition in micro-and macro-channels: An integrated mechanistic study," Combustion and Flame, Vol. 176, 2017, pp. 285-298.

[6] Radilescu, M. I., Sharpe, G. J., Law, C. K., and Lee, J., “The hydrodynamic structure of unstable cellular detonations,” Journal of Fluid Mechanics, Vol. 580, 2007, pp. 31-81.

[7] Starikovskiy, A., and Aleksandrov, N., "Plasma-assisted ignition and combustion," Progress in Energy and Combustion Science, Vol. 39, No. 1, 2013, pp. 61-110.

[8] Ju, Y., and Sun, W., "Plasma assisted combustion: Dynamics and chemistry," Progress in Energy and Combustion Science, Vol. 48, 2015, pp. 21-83.

[9] Starikovskaia, S. M., "Plasma-assisted ignition and combustion: nanosecond discharges and development of kinetic mechanisms," Journal of Physics D: Applied Physics, Vol. 47, No. 35, 2014, p. 353001.

[10] Adamovich, I. V., Choi, I., Jiang, N., Kim, J. H., Keshav, S., Lempert, W. R., Mintusov, E., Nishihara, M., Samimy, M., and Uddi, M., "Plasma assisted ignition and high-speed flow control: non-thermal and thermal effects," Plasma Sources Science and Technology, Vol. 18, No. 3, 2009, p. 034018.

[11] Kosarev, I. N., Aleksandrov, N. L., Kindysheva, S. V., Starikovskaia, S. M., and Starikovskii, A. Y., "Kinetics of ignition of saturated hydrocarbons by nonequilibrium plasma: CH4-containing mixtures," Combustion and Flame, Vol. 154, No. 3, 2008, pp. 569-586.

[12] Zhukov, V. P., Rakitin, A. E., and Starikovskii, A. Y., "Effect of high-voltage pulsed discharges on deflagration to detonation transition," Journal of Propulsion and Power, Vol. 24, No. 1, 2008, pp. 88-93.

[13] Gray, J. A. T., and Lacoste, D. A., "Enhancement of the transition to detonation of a turbulent hydrogen-air flame by nanosecond repetitively pulsed plasma discharges," Combustion and Flame, Vol. 199, 2019, pp. 258-266.

[14] Lepikhin, N. D., Popov, N. A., and Starikovskaia, S. M., "Fast gas heating and radial distribution of active species in nanosecond capillary discharge in pure nitrogen and N2: O2 mixtures," Plasma Sources Science and Technology, Vol. 27, No. 5, 2018, p. 055005 .

[15] Tropina, A., Mahamud, R., Yorn, D. W., and Miles, R. B., "Deflagration to detonation transition assisted by equilibrium and non-equilibrium plasma," AIAA Aviation 2019 Forum, 2019, p. 3119.

[16] Mc Bride, B., and Gordon, S., "NASA Computer Program for Calculation of Complex Chemical Equilibrium Compositions and Applications (CEA), https://www.grc.nasa.gov/WWW/CEAWeb/," NASA Reports 1311, Analysis (1994), and User's Manual and Program Description (1996), 1994.

[17] Bozhenkov, S. A., Starikovskaia, S. M., and Starikovskii, A. Y., "Nanosecond gas discharge ignition of H2- and CH4- containing mixtures," Combustion and flame, Vol. 133, No. 1-2, 2003, pp. 133-146. 